\title{
Impact of treatment with rosuvastatin and atorvastatin on cardiovascular outcomes: evidence from the Archimedes-simulated clinical
} trials

Furio Colivicchi'

Catarina Sternhufvud ${ }^{2}$ Sanjay K Gandhi ${ }^{3}$

'Cardiology Division, Emergency Department, San Filippo Neri Hospital, ASL Roma E, Rome, Italy; ${ }^{2}$ Global Payer Evidence and Pricing, AstraZeneca R\&D, Mölndal, Sweden; ${ }^{3}$ Global Health Economics and Outcomes Research, TEVA Pharmaceuticals, Frazer, PA, United States
Correspondence: Catarina Sternhufvud Global Payer Evidence and Pricing, AstraZeneca R\&D, Pepparedsleden I, 43I 53 Mölndal, Sweden

$\mathrm{Tel}+46317761000$

Email catarina.sternhufvud@astrazeneca. com
This article was published in the following Dove Press journal:

ClinicoEconomics and Outcomes Research

27 November 2015

Number of times this article has been viewed

Objective: No clinical trials have been conducted to directly compare the effect of the two high-intensity statins, rosuvastatin and atorvastatin, on cardiovascular outcomes. However, three such trials have been computer-simulated using the Archimedes model, an individualbased simulation of human physiology and behaviors, treatment interventions, and health care systems. The results are reviewed here.

Methods: The first simulated trial assessed clinical outcomes in patients receiving available doses of the two drugs. The second assessed the impact of initial treatment decisions, while the third assessed the effect of switching from rosuvastatin to atorvastatin.

Results: In the first simulated trial, treatment with rosuvastatin was estimated to result in greater reductions than treatment with atorvastatin in major adverse cardiac event (MACE) rates at 5 years and 20 years at all doses examined (relative risk [RR]: $0.897,0.888$, and 0.930 at 5 years for rosuvastatin $20 \mathrm{mg}$ vs atorvastatin $40 \mathrm{mg}$, rosuvastatin $40 \mathrm{mg}$ vs atorvastatin $80 \mathrm{mg}$, and rosuvastatin $20 \mathrm{mg}$ vs atorvastatin $80 \mathrm{mg}$, respectively; all $P<0.05$ ). In the second simulated trial, outcomes were significantly better in patients initially prescribed rosuvastatin than in those initially prescribed atorvastatin (RR of MACE at 5 years: $0.918 ; P<0.001$ ). In the third simulated trial, risk of MACE was significantly greater in patients switching from rosuvastatin to atorvastatin than in those remaining on rosuvastatin (RR at 5 years: $1.109 ; P<0.001$ ).

Conclusion: The results of these simulated clinical trials suggest improved outcomes among patients receiving rosuvastatin relative to patients receiving atorvastatin in various clinical settings.

Keywords: statins, rosuvastatin, atorvastatin, simulated clinical trials, outcome assessment

\section{Introduction}

According to World Health Organization statistics, ${ }^{1}$ cardiovascular disease (CVD) is the most common cause of mortality worldwide, being responsible for $\sim 30 \%$ of all deaths annually. Dyslipidemias, including elevated levels of total cholesterol, lowdensity lipoprotein cholesterol (LDL-C) and triglycerides, as well as low levels of high-density lipoprotein cholesterol (HDL-C), are strongly associated with increased cardiovascular risk. ${ }^{2-4}$ In a meta-analysis of 26 randomized controlled trials (RCTs) of statin therapy, including data from 169,138 individuals, reduction of LDL-C levels was shown to result in a $20 \%$ relative risk (RR) reduction in coronary mortality for every $1 \mathrm{mmol} / \mathrm{L}$ reduction in LDL-C (rate ratio: $0.80 ; 95 \%$ confidence interval [CI]: $0.74-0.87 ; P<0.0001) .{ }^{5}$ Despite the existence of effective treatment options, however, dyslipidemias remain prevalent in Europe. ${ }^{6-11}$ 
Statins (3-hydroxy-3-methyl-glutaryl coenzyme A reductase inhibitors) are the mainstay of therapy for reducing LDL-C levels. ${ }^{3,4,12}$ Several statins are available, differing in their absorption, bioavailability, plasma-protein binding, excretion, and solubility profiles. Statins may be classified according to dose into high-, moderate-, and lowintensity categories. ${ }^{3,12}$ High-intensity statins are defined in the guidelines of the American College of Cardiology/ American Heart Association (ACC/AHA) as those for which, on average, a daily dose reduces plasma LDL-C levels by $50 \%$ or more. According to this definition, high-intensity statins comprise rosuvastatin at a dose of $20-40 \mathrm{mg} / \mathrm{d}$ and atorvastatin at a dose of $40-80 \mathrm{mg} / \mathrm{d} .{ }^{12} \mathrm{High}$-intensity statin therapy has been shown to result in better cardiovascular outcomes than moderate-intensity therapy in patients with stable coronary heart disease or acute coronary syndrome (ACS). ${ }^{13-18}$ European and previous US guidelines recommend target plasma LDL-C concentrations, with the most aggressive LDL-C level targets recommended for patients at the highest risk of CVD. ${ }^{3,419}$ The most recent set of US guidelines recommends high-intensity statins for the treatment of all patients at high risk of CVD, regardless of their plasma LDL-C concentration. ${ }^{12}$

\section{Rosuvastatin and atorvastatin: existing clinical trial data}

Several clinical trials have demonstrated the efficacy of rosuvastatin and atorvastatin, as well as the superiority of high-intensity statin therapy over moderate- or low-intensity statin therapy, in the primary and secondary prevention of cardiovascular events. ${ }^{20}$ Approximately half of all myocardial infarctions and strokes occur in patients with LDL-C levels considered normal, ${ }^{21}$ and both rosuvastatin and atorvastatin have been shown to be more effective than placebo in reducing cardiovascular event rates in such individuals..$^{22,23}$ Justification for the Use of Statins in Primary Prevention: An Intervention Trial Evaluating Rosuvastatin (JUPITER) enrolled patients with LDL-C levels $<3.4 \mathrm{mmol} / \mathrm{L}$ and with C-reactive protein levels $>2 \mathrm{~g} / \mathrm{L}$. Treatment with rosuvastatin $20 \mathrm{mg} / \mathrm{d}$ resulted in a $44 \%$ reduction in the incidence of the first major cardiovascular event per 100 person-years of follow-up, relative to placebo (hazard ratio [HR]: 0.56; 95\% CI: $0.46-0.69 ; P<0.00001){ }^{22}$ The Anglo-Scandinavian Cardiac Outcomes Trial - Lipid Lowering Arm (ASCOT-LLA) enrolled patients with hypertension and total cholesterol levels $<6.5 \mathrm{mmol} / \mathrm{L}$ and randomized them to receive atorvastatin $10 \mathrm{mg} / \mathrm{d}$ or placebo. Atorvastatin reduced the incidence of the primary composite end point of myocardial infarction and cardiovascular death by $36 \%$ compared with placebo (HR: $0.64 ; 95 \%$ CI: $0.50-0.83 ; P=0.0005){ }^{23}$

In secondary prevention, atorvastatin $80 \mathrm{mg} / \mathrm{d}$ has been shown in the Treating to New Targets (TNT) study to be more effective than atorvastatin $10 \mathrm{mg} / \mathrm{d}$ in preventing cardiovascular events (HR: 0.78 ; 95\% CI: $0.69-0.89$; $P<0.001),{ }^{24}$ and in the Incremental Decrease in Endpoints through Aggressive Lipid Lowering (IDEAL) study to be more effective than simvastatin $20 \mathrm{mg}$ in reducing nonfatal acute myocardial infarctions (HR: 0.83 ; $95 \%$ CI: $0.71-0.98$; $P=0.02)^{17}$ in patients with stable coronary heart disease. Similarly, in individuals with ACS, atorvastatin $80 \mathrm{mg} / \mathrm{d}$ has been shown in the Myocardial Ischemia Reduction with Aggressive Cholesterol Lowering (MIRACL) trial to be more effective than placebo (RR: 0.84; 95\% CI: 0.70-1.00; $P=0.048)^{25}$ and in the Pravastatin or Atorvastatin Evaluation and Infection Therapy - Thrombolysis In Myocardial Infarction 22 (PROVE IT-TIMI 22) trial to be more effective than pravastatin $40 \mathrm{mg}$ (HR: 0.78 ; 95\% CI: $0.67-0.91 ; P=0.002)^{26}$ at preventing death and secondary cardiovascular events.

Rosuvastatin and atorvastatin have been compared headto-head in trials using surrogate end points to assess clinical outcomes. In the Comparison of the Efficacy and Safety of Rosuvastatin Versus Atorvastatin, Simvastatin, and Pravastatin Across Doses (STELLAR) study, across-dose comparisons showed that, in patients with LDL-C levels of $160-250 \mathrm{mg} / \mathrm{dL}$ and triglyceride levels $<400 \mathrm{mg} / \mathrm{dL}$, rosuvastatin $10-80 \mathrm{mg} / \mathrm{d}$ reduced LDL-C levels by a mean of $8.2 \%$ more than did atorvastatin $10-80 \mathrm{mg} / \mathrm{d}(P<0.001) .{ }^{27}$ Among patients with LDL-C levels of 3.4-5.7 mmol/L, rosuvastatin $10 \mathrm{mg} / \mathrm{d}$ was shown to be significantly more effective than atorvastatin $20 \mathrm{mg} / \mathrm{d}$ at reducing LDL-C levels (Prospective Study to Evaluate the Use of Low Doses of the Statins Atorvastatin and Rosuvastatin [PULSAR] $){ }^{28}$ Significantly more patients receiving rosuvastatin than those receiving atorvastatin achieved both the US National Cholesterol Education Program Adult Treatment Panel III (NCEP ATP III) and European LDL-C target levels. ${ }^{3,4,19,28}$ Treatment with either atorvastatin $80 \mathrm{mg} / \mathrm{d}$ or rosuvastatin $40 \mathrm{mg} / \mathrm{d}$ has been shown to be sustainable and results in reductions in atheroma volume. ${ }^{29}$

Despite the trials described above, however, significant evidence gaps remain in the literature with regard to the cardiovascular outcomes of intensive statin therapy. No trials have assessed cardiovascular event rates in patients prescribed rosuvastatin in the secondary prevention setting, and no trials have compared event rates directly in individuals receiving either rosuvastatin or atorvastatin. ${ }^{20}$ One potential solution to bridging this evidence gap is to simulate clinical 
trials using computerized models of patient physiology and treatment responses. This approach greatly reduces the time and cost required for implementing large, long-running outcomes trials in patients at risk of CVD.

\section{The Archimedes model}

The Archimedes model is an individual-based simulation of human pathophysiology and behaviors, treatment interventions, and health care systems. It is used to conduct virtual clinical trials and has demonstrated a high degree of accuracy. ${ }^{30-38}$ The model was originally developed to provide US decision makers with information about the outcomes that could be expected from clinical and administrative policies and programs, ${ }^{30,31}$ and is built using data from clinical trials, observational studies, and retrospective studies. ${ }^{32-34}$ Multiple diseases are included in the model, including obesity, metabolic syndrome, hypertension, dyslipidemia, CVD, diabetes mellitus and associated complications, nondiabetic retinopathy, chronic obstructive pulmonary disease, and cancers of the breast, colon, and lung.

The Archimedes model is continuously updated to incorporate the latest medical evidence and is unique in that it includes multiple levels of detail: epidemiological, clinical, and physiological. Each patient in a population is modeled as an individual, with unique risk factors, characteristics, and history of disease progression and treatment. Thus, the model represents the heterogeneity of a real population and enables analysis of multiple subpopulations. Virtual populations are created using a real population data set, typically that from the National Health and Nutrition Survey (NHANES), ${ }^{39}$ which is representative of the general US population.

The Archimedes model has been extensively validated, retrospectively against $>50$ clinical trials (full details of which can be found online ${ }^{34}$ ) and prospectively against 2 trials: the Diabetes Prevention Program (DPP) ${ }^{40}$ and the Collaborative Atorvastatin Diabetes Study (CARDS).$^{41}$ For CARDS, predictive validation was performed under formal supervision by two independent reviewers, and predictions were submitted in a sealed, dated envelope $>1$ month before the results were known to the principal investigators. The model accurately predicted the rates of myocardial infarction and stroke in individuals with diabetes mellitus, as well as the effects of atorvastatin on the incidence of CVD. ${ }^{32,33}$

\section{Archimedes-simulated clinical trials of rosuvastatin and atorvastatin}

The Archimedes model has been used to run three simulated clinical trials comparing rosuvastatin and atorvastatin.
The first simulated trial assessed clinical outcomes in patients prescribed varying available doses of the two drugs, ${ }^{42,43}$ while the second assessed the impact of initial treatment decisions in patients with dyslipidemia. ${ }^{44}$ The third assessed the impact of switching treatment from rosuvastatin to atorvastatin. ${ }^{45}$

To perform these virtual clinical trials, the core Archimedes model was expanded to include data on rosuvastatin $20 \mathrm{mg} / \mathrm{d}$ and $40 \mathrm{mg} / \mathrm{d}$ doses from the JUPITER and STELLAR trials, and data on atorvastatin $40 \mathrm{mg} / \mathrm{d}$ and $80 \mathrm{mg} / \mathrm{d}$ from the CARDS, ASCOT-LLA, STELLAR, and TNT trials. ${ }^{22-24,27,41}$ The effect of statins on clinical outcomes is modeled based on their effects on plasma lipid levels (primarily the total cholesterol to HDL-C ratio) and other pleiotropic effects, calibrated against trial results to capture additional benefits of treatment beyond those resulting directly from lipid biomarker changes. ${ }^{46}$

\section{Comparing the effectiveness of rosuvastatin and atorvastatin}

\begin{abstract}
Summary: A simulated trial was run to compare the effects of rosuvastatin and atorvastatin treatment over 20 years. The primary outcome was first major adverse cardiovascular event (MACE). Rosuvastatin was estimated to result in significantly greater reductions in MACE than treatment with atorvastatin.
\end{abstract}

In the first of the three virtual clinical trials of rosuvastatin and atorvastatin, outcomes were assessed in patients receiving varying available doses of each drug. ${ }^{42,43} \mathrm{~A}$ virtual population was created, based on the NHANES 1999-2006 data set, of individuals aged 45-70 years with a 10-year cardiovascular Framingham Risk Score (FRS) of at least 5\% $(\mathrm{N}=55,000) .{ }^{47}$ Subpopulations were also assessed: those with an FRS of $5 \%-10 \%(n=21,902), 10 \%-20 \% \quad(n=23,908)$, or $>20 \%$ $(n=9,190)$; those with a European Systematic Coronary Risk Evaluation (SCORE) of at least $5 \%(n=16,687)$ or $>10 \%$ $(n=5,065)$; those with diabetes mellitus $(n=10,301)$; and those in the secondary prevention setting with a previous diagnosis of myocardial infarction or stroke $(n=3,060)$.

The simulated trial included a washout period during which previous lipid-lowering therapies were discontinued, following which patients were run through a series of trial arms in each of which they were treated with one of the target therapies. The same simulated individuals were run through the different rosuvastatin and atorvastatin treatment arms to improve comparability and to isolate treatment effects. Compliance with prescribed therapy was assumed to be $100 \%$, and treatment of other cardiovascular risk factors was 
modeled according to guideline recommendations, ${ }^{48,49}$ with compliance with guidelines calibrated against that observed in the NHANES data set. The primary outcome of the study was first MACE, defined as the first occurrence of fatal or nonfatal myocardial infarction/stroke or cardiovascular death.

At 1 year, mean reductions in the total cholesterol to HDL-C ratio in the population with an FRS of at least 5\% were $43 \%$ for rosuvastatin $20 \mathrm{mg} / \mathrm{d}, 45 \%$ for rosuvastatin $40 \mathrm{mg} / \mathrm{d}, 38 \%$ for atorvastatin $40 \mathrm{mg} / \mathrm{d}$, and $41 \%$ for atorvastatin $80 \mathrm{mg} / \mathrm{d}$. These reductions were in line with results from the STELLAR trial. ${ }^{27}$ In the same population, mean reductions in LDL-C levels were $53 \%$ for rosuvastatin $20 \mathrm{mg} / \mathrm{d}$, $56 \%$ for rosuvastatin $40 \mathrm{mg} / \mathrm{d}, 49 \%$ for atorvastatin $40 \mathrm{mg} / \mathrm{d}$, and $53 \%$ for atorvastatin $80 \mathrm{mg} / \mathrm{d}$.

Rosuvastatin $20 \mathrm{mg} / \mathrm{d}$ significantly improved outcomes in the overall population with an FRS of at least $5 \%$ at 5 years, relative to atorvastatin $40 \mathrm{mg} / \mathrm{d}$ (RR of MACE: $0.897 ; 95 \% \mathrm{CI}$ : 0.884-0.911). The RR was approximately similar at 20 years, and in each of the subpopulations analyzed, with a slight decrease in benefit for the higher-risk groups. Comparing rosuvastatin $40 \mathrm{mg} / \mathrm{d}$ with atorvastatin $80 \mathrm{mg} / \mathrm{d}$, the RR of MACE at 5 years was 0.888 (95\% CI: 0.869-0.906); while comparing rosuvastatin $20 \mathrm{mg} / \mathrm{d}$ with atorvastatin $80 \mathrm{mg} / \mathrm{d}$, the RR of MACE at 5 years was 0.930 (95\% CI: 0.916-0.943). ${ }^{43}$

The number needed to treat (NNT) with rosuvastatin relative to the active comparator atorvastatin to prevent one incidence of MACE at 5 years, in the overall population with an FRS of at least 5\%, was 268 for rosuvastatin $20 \mathrm{mg} / \mathrm{d}$ vs atorvastatin $40 \mathrm{mg} / \mathrm{d}, 254$ for rosuvastatin $40 \mathrm{mg} / \mathrm{d}$ relative to atorvastatin $80 \mathrm{mg} / \mathrm{d}$, and 408 for rosuvastatin $20 \mathrm{mg} / \mathrm{d}$ vs atorvastatin $80 \mathrm{mg} / \mathrm{d}$ (Figure 1). NNT values decreased as risk increased, and in the secondary prevention population, NNT values were 70, 63, and 137 for rosuvastatin $20 \mathrm{mg} / \mathrm{d}$ vs atorvastatin $40 \mathrm{mg} / \mathrm{d}$, rosuvastatin $40 \mathrm{mg} / \mathrm{d}$ vs atorvastatin $80 \mathrm{mg} / \mathrm{d}$, and rosuvastatin $20 \mathrm{mg} / \mathrm{d}$ vs atorvastatin $80 \mathrm{mg} / \mathrm{d}$, respectively.

\section{Impact of initial statin treatment decisions}

Summary: A second simulated trial assessed the effects of initiating statin therapy with either rosuvastatin or atorvastatin, and intensifying treatment as necessary. Risk of MACE was estimated to be significantly reduced over 10 years in patients initially prescribed rosuvastatin relative to those initially prescribed atorvastatin.

The second of the three virtual clinical trials assessed the impact of starting patients on statin therapies of different intensities and titrating treatment until LDL-C target levels were reached ${ }^{44}$ In this trial, the virtual population consisted of patients aged 45-70 years who had LDL-C levels exceeding conservative US ATP-III targets ${ }^{19}$ after a washout period during which no lipid-lowering drugs were received $(\mathrm{N}=50,025)$. Subpopulations were also assessed, consisting of patients with diabetes mellitus, those with a history of myocardial infarction or stroke, those with LDL-C levels more than double their aggressive ATP-III targets, those classified as being at moderate cardiovascular risk according to standard ATP-III guidelines, and those classified as being at high cardiovascular risk according to standard ATP-III guidelines. ${ }^{19}$

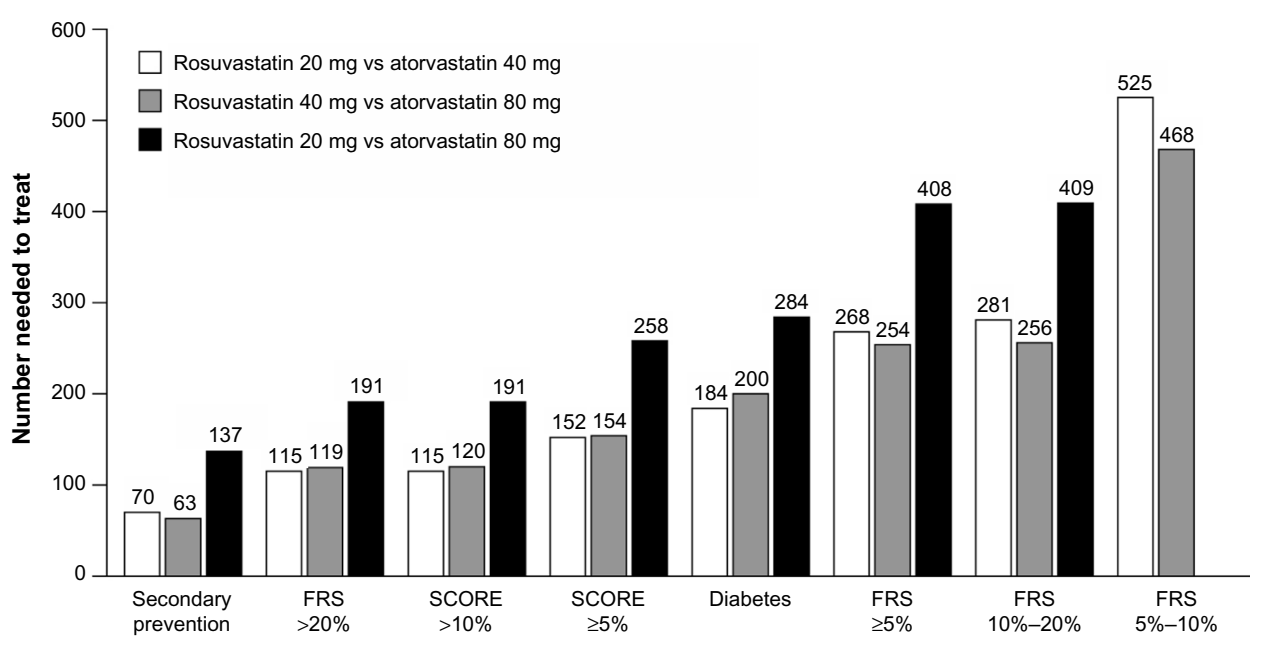

Figure I Number needed to treat to prevent one incidence of MACE at 5 years by treatment with rosuvastatin relative to treatment with atorvastatin, according to risk group. Notes: Smaller values indicate greater benefit. For the FRS 5\%-10\% group, no analysis was performed comparing rosuvastatin 20 mg with atorvastatin 80 mg. Adapted from Schuetz CA, van Herick A, Alperin P, Peskin B, Hsia J, Gandhi S. Comparing the effectiveness of rosuvastatin and atorvastatin in preventing cardiovascular outcomes: estimates using the Archimedes model. J Med Econ. 20I2; I5(6): I I 18-II29.42 Copyright @ 20I5. Adapted with permission of the author and publisher (Taylor and Francis Ltd, http://www.tandfonline.com).

Abbreviations: FRS, Framingham Risk Score; MACE, major adverse cardiovascular event; SCORE, Systematic Coronary Risk Evaluation. 
The trial used a multiarm design, in which, after the washout period, patients began treatment with either atorvastatin $(10 \mathrm{mg} / \mathrm{d}, 20 \mathrm{mg} / \mathrm{d}, 40 \mathrm{mg} / \mathrm{d}$, or $80 \mathrm{mg} / \mathrm{d})$ or rosuvastatin $(10 \mathrm{mg} / \mathrm{d}, 20 \mathrm{mg} / \mathrm{d}$, or $40 \mathrm{mg} / \mathrm{d})$. A simvastatin treatment arm was also included. As in the first simulated clinical trial, the same simulated patient population was used in each arm of the study. Within each arm, the proportion of individuals initiating treatment at each dose was calibrated to US pharmacy claims data using an algorithm that minimized overtreatment. Patient compliance was calibrated to produce ATP-III target LDL-C level attainment rates consistent with those from previous publications..$^{50,51}$

In follow-up visits for the first 5 years ( 6 weeks after the start of the trial, and periodically thereafter at frequencies ranging from 5 months to 1 year, consistent with ATP-III guidelines), patients were eligible for treatment intensification if their LDL-C levels were still above target. Those eligible for intensification could remain on their current treatment, increase the dose of their currently prescribed statin if not already at the maximum dose, or switch from atorvastatin to rosuvastatin $10 \mathrm{mg} / \mathrm{d}, 20 \mathrm{mg} / \mathrm{d}$, or $40 \mathrm{mg} / \mathrm{d}$. The assignment of each eligible patient to a different intensification option was randomized, with proportions of individuals intensifying treatment calibrated at Year 1 against pharmacy claims data. For example, for patients initially prescribed simvastatin $20 \mathrm{mg} / \mathrm{d}, 13 \%$ increased their simvastatin dose and $1 \%$ switched to rosuvastatin at 1 year, exactly in line with claims data. After 5 years, no further intensification was offered, and patients remained on their current statin for a further 5 years until the end of the simulation. Treatment of other cardiovascular risk factors was modeled according to guideline recommendations, with compliance with guidelines calibrated against that observed in the NHANES data set. ${ }^{48,49}$ The primary end point of the trial was MACE, as defined in the first simulated trial. ${ }^{42}$

After 1 year, mean reductions in LDL-C concentrations were $34 \%$ and $38 \%$ for those initiating treatment with atorvastatin and rosuvastatin, respectively. The corresponding proportions of patients meeting their conservative ATP-III target LDL-C levels were $69 \%$ and $73 \%$. For the primary end point, the RR of MACE at 5 years was 0.918 (95\% CI: $0.898-0.938 ; P<0.001)$ for initial treatment with rosuvastatin relative to atorvastatin (Figure 2). At 10 years, the corresponding RR was 0.919 (95\% CI: 0.906-0.932; $P<0.001)$. RRs were approximately similar in all subgroups analyzed, with a slight decrease in benefit for higher-risk groups. The NNT to prevent one incidence of MACE was 275 for initial treatment with rosuvastatin relative to atorvastatin

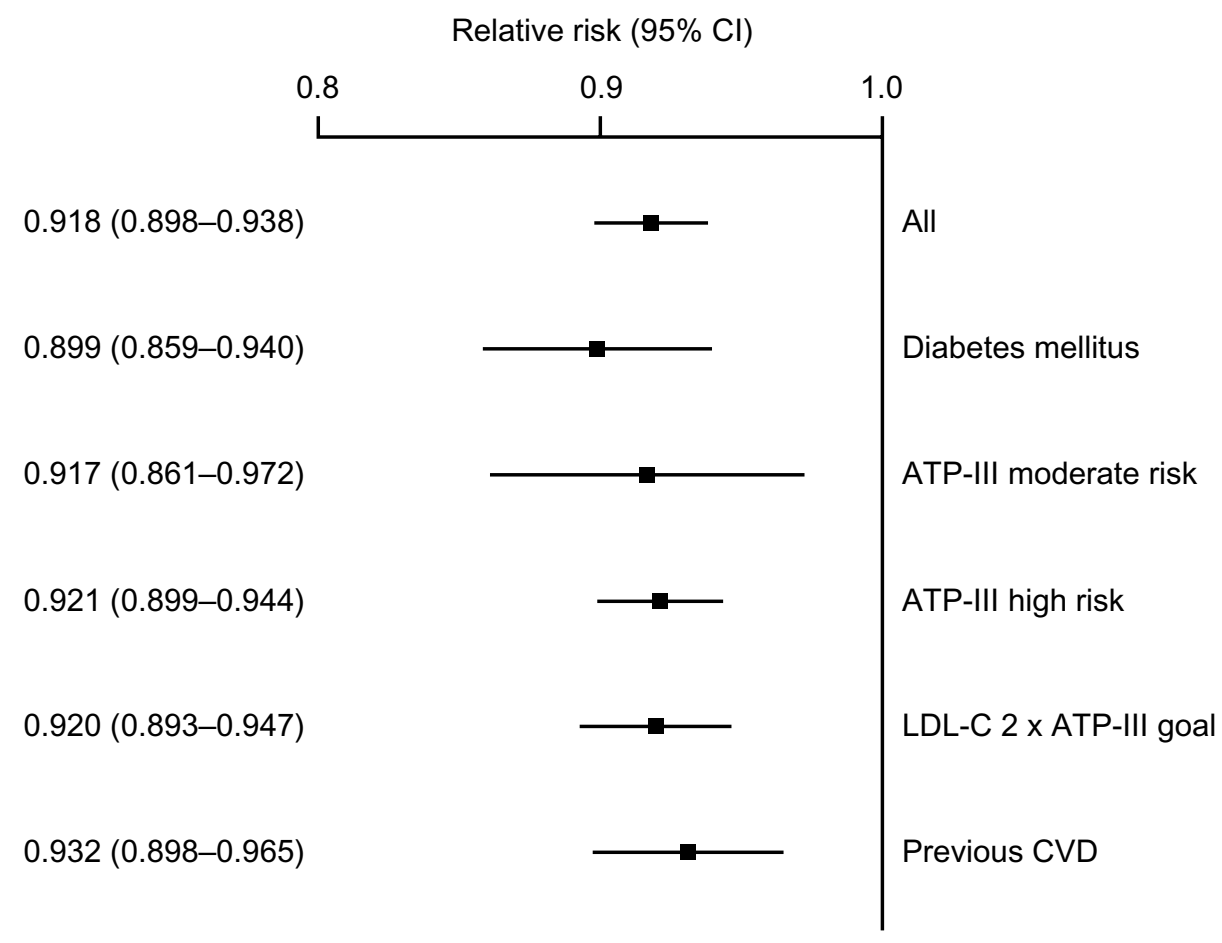

Figure 2 Five-year relative risk of MACE for initial treatment with rosuvastatin relative to atorvastatin, separated according to risk group.

Note: Adapted from van Herick A, Schuetz CA, Alperin P, Bullano MF, Balu S, Gandhi S. The impact of initial statin treatment decisions on cardiovascular outcomes in clinical care settings: estimates using the Archimedes model. Clinicoecon Outcomes Res. 2012;4:337-347. ${ }^{44}$ Copyright (C 20I2; permission conveyed through Copyright Clearance Center, Inc. Abbreviations: ATP, adult treatment panel; Cl, confidence interval; CVD, cardiovascular disease; LDL-C, low-density lipoprotein cholesterol; MACE, major adverse cardiovascular event. 
at 5 years and 135 at 10 years. NNT values decreased as risk increased, with the lowest numbers observed in the subgroup with previous CVD (NNT 105 at 5 years).

\section{Impact of switching treatment from rosuvastatin to atorvastatin}

Summary: A third simulated trial assessed the effects of initiating therapy with rouvastatin, and switching to atorvastatin after 6 weeks. The cumulative incidence of MACE over 10 years was estimated to be higher among patients switching to atorvastatin than among patients remaining on rosuvastatin.

In the third simulated clinical trial, the effect of switching patients from rosuvastatin to atorvastatin was assessed. ${ }^{45}$ In this trial, the virtual population was created based on the NHANES 1999-2008 data set and consisted of patients aged 45-70 years who had LDL-C levels exceeding conservative US ATP-III targets ${ }^{19}$ after a washout period during which no lipid-lowering drugs were received $(\mathrm{N}=50,038)$. Subgroups assessed included patients with diabetes mellitus, those with a history of myocardial infarction or stroke, those with LDL-C levels more than double their aggressive ATP-III targets, those classified as being at moderate cardiovascular risk according to standard ATP-III guidelines, those classified as being at high cardiovascular risk according to standard ATP-III guidelines, and those whose LDL-C levels exceeded ATP-III goals after initial statin therapy. ${ }^{19}$

After the initial washout period, all patients were prescribed rosuvastatin at doses of $10 \mathrm{mg} / \mathrm{d}, 20 \mathrm{mg} / \mathrm{d}$, or $40 \mathrm{mg} / \mathrm{d}$, matching pharmacy claims data and minimizing overtreatment. Overall, 74\% received rosuvastatin $10 \mathrm{mg} / \mathrm{d}$, while $23 \%$ received $20 \mathrm{mg} / \mathrm{d}$ and $3 \%$ received $40 \mathrm{mg} / \mathrm{d}$. Compliance was assumed to be $100 \%$. After 6 weeks, patients had a follow-up visit at which participants in the experimental arm were switched to atorvastatin at twice their original rosuvastatin dose, while those in the control arm remained on rosuvastatin. The same population was used in both arms of the trial. Additionally, patients for whom LDL-C levels remained above ATP-III targets were eligible for intensification of either rosuvastatin or atorvastatin therapy, according to the arm of the trial that they were in. As in the trial assessing the effects of initial treatment decisions, patients continued to have follow-up visits at varying frequencies for 5 years, during which treatment could be intensified if LDL-C levels remained above target. After 5 years, no further intensification was offered, and patients remained on their current treatment until the end of the simulation at 10 years. Treatment of other cardiovascular risk factors was modeled according to guideline recommendations, with compliance with guidelines calibrated against that observed in the NHANES data set. ${ }^{48,49}$ The primary outcome of the trial was MACE, as defined previously.

The overall reduction in patients' LDL-C levels was $48 \%$ at 1 year and $47 \%$ at 5 years when individuals remained on rosuvastatin, with the reduction being $43 \%$ at both 1 year and 5 years when patients were switched to atorvastatin. ATP-III LDL-C target levels were attained by $91 \%$ of those remaining on rosuvastatin and $87 \%$ of those switching to atorvastatin, at both 1 year and 5 years. The cumulative incidence of MACE was higher in patients who switched to atorvastatin than in those who remained on rosuvastatin (Figure 3). At 5 years, the RR of MACE was 1.109 (95\% CI: 1.092-1.127; $P<0.001)$ for patients who switched to atorvastatin relative to those who remained on rosuvastatin. At 10 years, the corresponding RR was 1.115 (95\% CI: $1.103-1.127 ; P<0.001)$. The number needed to harm $(\mathrm{NNH})$ resulting in one extra MACE was 262 at 5 years and 117 at 10 years, for patients who switched to atorvastatin relative to those who remained on rosuvastatin. RRs were similar across all subpopulations assessed, and NNHs were lower among higher-risk subgroups. The NNH at 5 years among those with previous CVD was 126 for patients switching to atorvastatin relative to those remaining on rosuvastatin.

\section{Results in clinical context}

The simulated clinical trials of rosuvastatin and atorvastatin described above have filled an important gap in the literature, comparing the effectiveness of these two high-intensity statins in reducing the incidence of cardiovascular events. On the basis of the simulated trials, greater efficacy has been estimated for rosuvastatin than for atorvastatin in improving cardiovascular outcomes at all doses and in all risk groups tested. In the overall population, in the first simulated trial, the NNT to prevent one incidence of MACE in direct dose comparisons was 268 for rosuvastatin $20 \mathrm{mg} / \mathrm{d}$ vs atorvastatin $40 \mathrm{mg} / \mathrm{d}, 254$ for rosuvastatin $40 \mathrm{mg} / \mathrm{d}$ relative to atorvastatin $80 \mathrm{mg} / \mathrm{d}$, and 408 for rosuvastatin $20 \mathrm{mg} / \mathrm{d} \mathrm{vs}$ atorvastatin $80 \mathrm{mg} / \mathrm{d}$. The LDL-C-lowering effectiveness ratio for rosuvastatin compared with atorvastatin was $4: 1$, given the similar molecular weights of the two compounds; a $20 \mathrm{mg} / \mathrm{d}$ dose of rosuvastatin had an effect similar to that of an $80 \mathrm{mg} / \mathrm{d}$ dose of atorvastatin. The $20 \mathrm{mg} / \mathrm{d}$ dose of rosuvastatin reduced mean LDL-C levels by $>50 \%$ from the baseline. These results are in line with data from the STELLAR and PULSAR trials showing that rosuvastatin is more effective 


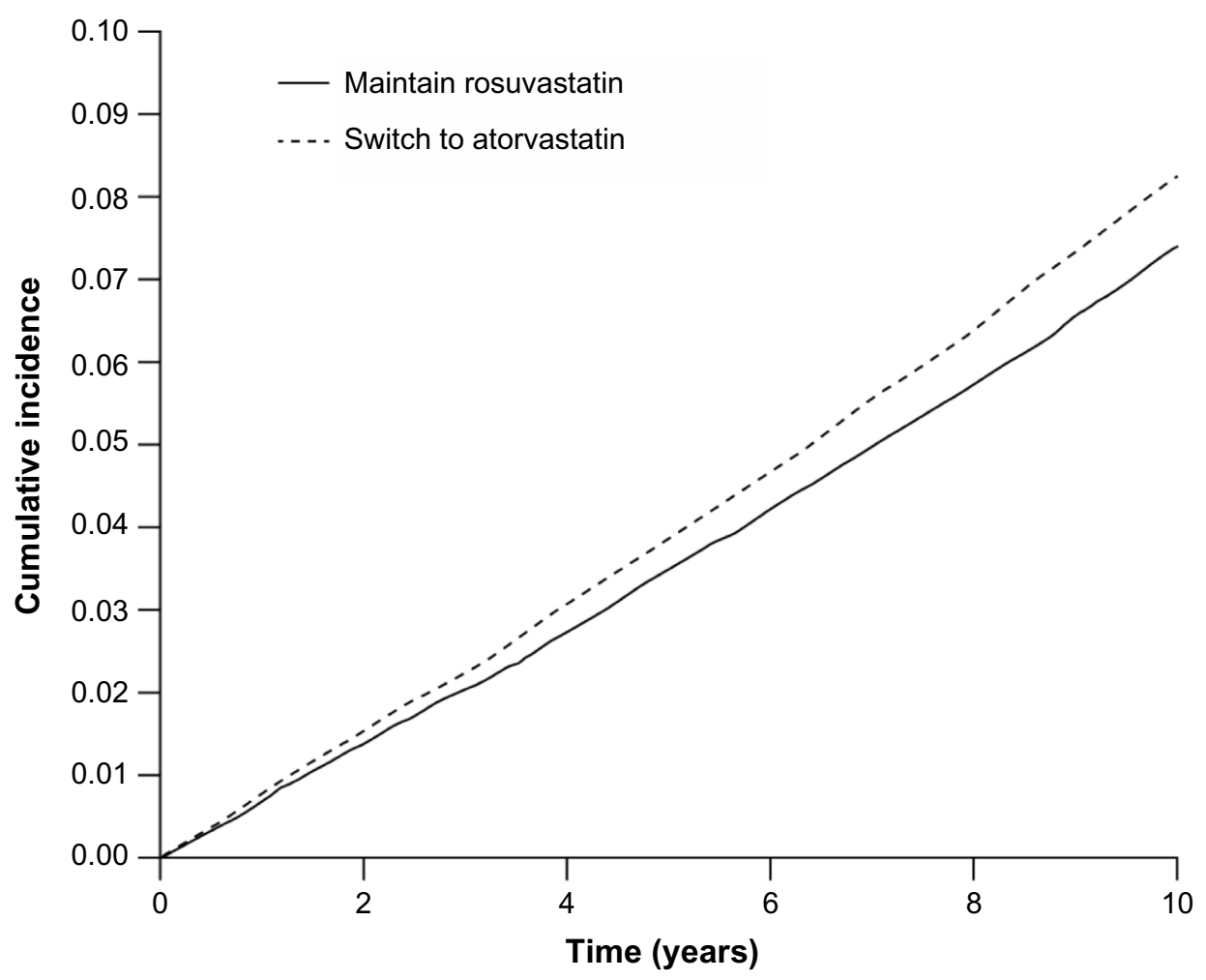

Figure 3 Kaplan-Meier cumulative incidence of MACE for patients who switch from rosuvastatin to atorvastatin (dotted line) and for patients who remain on rosuvastatin (solid line).

Note: Adapted from Folse H, Sternhufvud C, Andy Schuetz C, Rengarajan B, Gandhi S. Impact of switching treatment from rosuvastatin to atorvastatin on rates of cardiovascular events. Clin Ther. 20|4;36(I):58-69.45

Abbreviation: MACE, major adverse cardiovascular event.

than atorvastatin at reducing patients' LDL-C levels. ${ }^{27,28}$ The benefits of beginning treatment with an appropriate-intensity statin therapy have also been demonstrated, in comparison with starting therapy with a lower-intensity statin and titrating until LDL-C targets are met.

Many previous studies have shown that patients with dyslipidemia are often undertreated in clinical practice; the 20062007 European Action on Secondary and Primary Prevention through Intervention to Reduce Events (EUROASPIRE) III survey conducted in 22 European countries found that $79.8 \%$ of patients with coronary heart disease were receiving lipid-lowering therapy, varying across countries from $41.6 \%$ (Lithuania) to $95.4 \%$ (Finland). ${ }^{52} \mathrm{~A}$ total of $51.1 \%$ of patients had elevated total cholesterol levels ( $\geq 4.5 \mathrm{mmol} / \mathrm{L})$, while $54.5 \%$ had raised LDL-C levels ( $\geq 2.5 \mathrm{mmol} / \mathrm{L}$ ), $36.7 \%$ had low HDL-C levels $(<1.0 \mathrm{mmol} / \mathrm{L}$ for men and $<1.2 \mathrm{mmol} / \mathrm{L}$ for women), and $34.7 \%$ had increased triglyceride levels $(\geq 1.7 \mathrm{mmol} / \mathrm{L}){ }^{52}$ Thus, the treatment of dyslipidemia in these patients was inadequate. The use of lipid-lowering therapy has, however, increased considerably since the first EUROASPIRE survey conducted in 1995-1996. ${ }^{53}$

Similarly, the Centralized Pan-European Survey on the Under-treatment of Hypercholesterolaemia (CEPHEUS) has found that, in eight European countries, achievement of LDL-C target levels among patients who had received lipid-lowering therapy for at least 3 months was only $55.3 \%,{ }^{54}$ whereas the European Study on Cardiovascular Risk Prevention and Management in Usual Daily Practice (EURIKA) showed that across 12 European countries, among treated patients with dyslipidemia, only $41.2 \%$ attained both their total cholesterol and LDL-C targets. An Italian nationwide survey has also yielded similar evidence of inadequate lipid control. ${ }^{55}$ Among 878 consecutive patients who suffered a cardiovascular event requiring hospitalization, LDL-C was $<100 \mathrm{mg} / \mathrm{dL}$ in $\sim 57 \%$ and $<70 \mathrm{mg} / \mathrm{dL}$ in only $20 \%$ of patients.

Several studies have also demonstrated that, while most physicians agree with guideline recommendations for the reduction of cardiovascular risk, they do not follow them aggressively enough. ${ }^{56,57}$ Prescribed statin doses are often too low. ${ }^{58-61}$ Titration rates from low-intensity statins to higher-intensity treatment are also low according to several studies, ${ }^{44,62,63}$ in addition to the pharmacy claims data from the US against which the Archimedes-simulated study was calibrated.

It has therefore been suggested that an improved strategy for statin treatment would be to start patients on an 
intensity of statin therapy appropriate to their global level of cardiovascular risk. ${ }^{64}$ Multiple studies have demonstrated improved goal achievement in patients treated using such an approach, and the results of the Archimedes-simulated study, showing improved clinical outcomes in patients treated aggressively from the start of their therapy, are in agreement with this. ${ }^{60,64-68}$ The approach is also in line with recommendations in the $2013 \mathrm{ACC} / \mathrm{AHA}$ guidelines on the treatment of blood cholesterol, which recommend moderateor high-intensity statin therapy for patients in four stratified risk groups (individuals with clinical atherosclerotic CVD [ASCVD], individuals with LDL-C levels $\geq 190 \mathrm{mg} / \mathrm{dL}$, individuals aged 40-75 years with diabetes mellitus who have LDL-C levels of 70-189 mg/dL, and individuals without clinical ASCVD or diabetes mellitus who are 40-75 years of age with LDL-C levels of 70-189 mg/dL and an estimated 10 -year ASCVD risk of $\geq 7.5 \%) .{ }^{12}$ In a retrospective study conducted in the US, ${ }^{69}$ it has been shown that lower statin titration rates are required for patients initiated on rosuvastatin treatment than for those in whom treatment with other statins has been initiated.

In the third simulated clinical trial, an increased cardiovascular risk was demonstrated for patients who switched from rosuvastatin to atorvastatin relative to those who did not switch treatments. A process of switching from rosuvastatin to atorvastatin may occur, now that generic atorvastatin has become available while the patent on rosuvastatin remains in place. Several previous studies have demonstrated that such switching can occur; for instance, in Germany, when the introduction of a reference pricing system resulted in the requirement for patient co-payments for the prescription of atorvastatin relative to generic statins, individuals previously prescribed atorvastatin showed higher rates of nonadherence and discontinuation of treatment relative to patients previously prescribed other statins that were unaffected by the reference pricing system $(P<0.0001){ }^{70}$

Similarly, in Norway, $40 \%$ of patients prescribed atorvastatin switched to generic simvastatin after reimbursement regulations were introduced that encouraged this. ${ }^{71}$ In Iceland, when regulations were introduced that meant that for the majority of patients only simvastatin $10 \mathrm{mg} / \mathrm{d}$ or $20 \mathrm{mg} / \mathrm{d}$ could be reimbursed, rates of cholesterol-level goal attainment were significantly reduced. ${ }^{72}$ Mean plasma concentrations of both total cholesterol and LDL-C were increased. Switching from high-intensity to moderate- or low-intensity statin treatments has been shown to result in significantly lower levels of LDL-C-level goal attainment. ${ }^{18,73}$ Among patients with ACS who were prescribed atorvastatin
$80 \mathrm{mg} / \mathrm{d}$ on hospital discharge and who either switched to a moderate-intensity statin or remained on their initial therapy, treatment switching was an independent predictor of adverse clinical outcomes (HR: 2.7; 95\% CI: $1.7-5.1 ; P=0.004) .{ }^{18}$

\section{Limitations of the Archimedes model}

The simulated trials described were all conducted using a mathematical model, and as such, are subject to dependence on the evidence used to produce it. For statin treatment, this included the results of CARDS, ASCOT, JUPITER, and the TNT trial. Uncertainty in the true treatment effects of statins, reflected in the $95 \%$ CIs of these studies, propagates into the results of the simulated trials. A further significant limitation is the fact that the model does not include adverse events related to statin treatment. As a result of this, it is not possible to comment on the estimated occurrence of adverse events in the different treatment arms of each trial. It was also assumed that compliance to the prescribed treatment in each trial was $100 \%$. Although this enables the pharmacologic treatment effect of statins to be modeled, it does not take into account reductions in adherence to treatment over time that occur in the real world, which can be as high as $50 \%$ within the 1 st year of treatment. ${ }^{74}$ Analysis of pharmaceutical claims data during calibration of the model also revealed a small but significant proportion of individuals reducing their initially prescribed statin treatment intensity. The effects of statin therapy have also been assumed to remain constant over the duration of the trials, but the trial results used to create the model had durations of less than the 5- to 20-year timescale of the simulated trials. In addition, the three end points included in the definition of MACE, namely, myocardial infarction, stroke, and cardiovascular death, do not all reflect the same disease process or effect of statin treatment. However, they are hard clinical end points of direct importance to patients and have thus been assessed as a composite end point.

\section{Conclusion: clinical perspective}

The results of the Archimedes-simulated clinical trials are clinically meaningful, with implications for statin prescription practices. The results highlight the greater estimated efficacy of rosuvastatin relative to atorvastatin in reducing the occurrence of cardiovascular events in various clinical settings. According to the European Society of Cardiology/European Atherosclerosis Society (ESC/EAS) guidelines on CVD prevention, patients requiring the most aggressive statin therapy include all those defined as being at very high cardiovascular risk: those with established CVD, those with type 1 or type 
2 diabetes mellitus with target organ damage, those with moderate-to-severe chronic kidney disease, and those with a SCORE of at least $5 \% .{ }^{4,75}$ According to the ACC/AHA guidelines, patients who should be prescribed a high-intensity statin include those with established ASCVD, those with LDL-C levels $>190 \mathrm{mg} / \mathrm{dL}$, and those with type 1 or type 2 diabetes mellitus with an estimated 10-year risk of ASCVD of at least $7.5 \%$. The results from the simulated trials suggest that rosuvastatin should be preferred to atorvastatin as the high-intensity statin therapy for such patients.

Although RCTs remain the gold standard in evidencebased medicine, the cost and time required to conduct them often means that they are not feasible. When RCT evidence is missing but is needed to inform best clinical practice, simulated clinical trials can provide valuable insight into the likely outcomes of treatment variation. The Archimedes model used in the simulated trials described in this review has been extensively validated both retrospectively and prospectively, demonstrating its applicability to real-world clinical practice. ${ }^{32-34}$

\section{Acknowledgment}

Medical writing support, funded by AstraZeneca R\&D, Mölndal, Sweden, was provided by Stephen Sweet $\mathrm{PhD}$ from Oxford PharmaGenesis.

\section{Disclosure}

Funding for the Archimedes-simulated clinical trials was provided by AstraZeneca. Furio Colivicchi reports receiving research funding from AstraZeneca, Bayer, Boehringer Ingelheim, and Pfizer; speaker fees from AstraZeneca, Bayer, Boehringer Ingelheim, Menarini, Merck Sharp and Dohme, and Pfizer; and serving on advisory boards for Aegerion, AstraZeneca, Bayer, Merck Sharp and Dohme, and Pfizer. Catarina Sternhufvud is an employee of AstraZeneca. Sanjay $\mathrm{K}$ Gandhi was an employee of AstraZeneca at the time these simulated clinical trials were carried out. The authors report no other conflicts of interest in this work.

\section{References}

1. WHO. Global Status Report on Noncommunicable Diseases 2010. 2011. Available from: http://whqlibdoc.who.int/publications/2011/ 9789240686458_eng.pdf.

2. Baigent C, Keech A, Kearney PM, et al; Cholesterol Treatment Trialists' (CTT) Collaborators. Efficacy and safety of cholesterol-lowering treatment: prospective meta-analysis of data from 90,056 participants in 14 randomised trials of statins. Lancet. 2005;366(9493):1267-1278.

3. Reiner Z, Catapano AL, De Backer G, et al; European Association for Cardiovascular Prevention and Rehabilitation. ESC/EAS Guidelines for the management of dyslipidaemias: the task force for the management of dyslipidaemias of the European society of cardiology (ESC) and the European atherosclerosis society (EAS). Eur Heart J. 2011;32(14): 1769-1818.
4. Perk J, De Backer G, Gohlke H, et al. European guidelines on cardiovascular disease prevention in clinical practice (version 2012). The fifth joint task force of the european society of cardiology and other societies on cardiovascular disease prevention in clinical practice (constituted by representatives of nine societies and by invited experts). Eur Heart J. 2012;33(13):1635-1701.

5. Cholesterol Treatment Trialists' (CTT) Collaboration, Baigent C, Blackwell L, et al. Efficacy and safety of more intensive lowering of LDL cholesterol: a meta-analysis of data from 170,000 participants in 26 randomised trials. Lancet. 2010;376(9753):1670-1681.

6. Laccetti R, Pota A, Stranges S, et al. Evidence on the prevalence and geographic distribution of major cardiovascular risk factors in Italy. Public Health Nutr. 2012;16(2):305-315.

7. Grassi G, Arenare F, Dell'oro R, et al. Prevalence of cardiovascular risk factors in an unselected Italian population. Results of the Cardiolab Project 2004-2008. Acta Cardiol. 2009;64(6):771-778.

8. Gomez-Huelgas R, Mancera-Romero J, Bernal-Lopez MR, et al. Prevalence of cardiovascular risk factors in an urban adult population from southern Spain. IMAP study. Int J Clin Pract. 2011;65(1): 35-40.

9. Gabriel R, Alonso M, Segura A, et al; ERICE Cooperative Group. Prevalence, geographic distribution and geographic variability of major cardiovascular risk factors in Spain. Pooled analysis of data from population-based epidemiological studies: the ERICE study. Rev Esp Cardiol. 2008;61(10):1030-1040.

10. Firmann M, Marques-Vidal P, Paccaud F, et al. Prevalence, treatment and control of dyslipidaemia in Switzerland: still a long way to go. Eur J Cardiovasc Prev Rehabil. 2010;17(6):682-687.

11. Guallar-Castillón P, Gil-Montero M, León-Muñoz LM, et al. Magnitude and management of hypercholesterolemia in the adult population of Spain, 2008-2010: the ENRICA study. Rev Esp Cardiol (Engl ED). 2012;65(6):551-558.

12. Stone NJ, Robinson J, Lichtenstein AH, et al. 2013 ACC/AHA guideline on the treatment of blood cholesterol to reduce atherosclerotic cardiovascular risk in adults: a report of the American College of Cardiology/ American heart association task force on practice guidelines. Circulation. 2014;129(25 Suppl 2):S1-S45.

13. Cannon CP, Steinberg BA, Murphy SA, Mega JL, Braunwald E. Metaanalysis of cardiovascular outcomes trials comparing intensive versus moderate statin therapy. J Am Coll Cardiol. 2006;48(3):438-445.

14. Cannon CP, Braunwald E, McCabe CH, et al; Pravastatin or Atorvastatin Evaluation and Infection Therapy-Thrombolysis in Myocardial Infarction 22 Investigators. Intensive versus moderate lipid lowering with statins after acute coronary syndromes. $N$ Engl J Med. 2004; 350(15):1495-1504.

15. LaRosa JC, Grundy SM, Waters DD, et al; Treating to New Targets (TNT) Investigators. Intensive lipid lowering with atorvastatin in patients with stable coronary disease. N Engl J Med. 2005;352(14):1425-1435.

16. de Lemos JA, Blazing MA, Wiviott SD, et al; Investigators. Early intensive vs a delayed conservative simvastatin strategy in patients with acute coronary syndromes: phase $\mathrm{Z}$ of the $\mathrm{A}$ to $\mathrm{Z}$ trial. JAMA. 2004; 292(11):1307-1316.

17. Pedersen TR, Faergeman O, Kastelein JJ, et al; Incremental Decrease in End Points Through Aggressive Lipid Lowering (IDEAL) Study Group. High-dose atorvastatin vs usual-dose simvastatin for secondary prevention after myocardial infarction: the IDEAL study: a randomized controlled trial. JAMA. 2005;294(19):2437-2445.

18. Colivicchi F, Tubaro M, Santini M. Clinical implications of switching from intensive to moderate statin therapy after acute coronary syndromes. Int $J$ Cardiol. 2011;152(1):56-60.

19. National Cholesterol Education Program. Third report of the national cholesterol education program (NCEP) expert panel on detection, evaluation, and treatment of high blood cholesterol in adults (adult treatment panel III) final report. Circulation. 2002;106(25):3143-3421.

20. Luvai A, Mbagaya W, Hall AS, Barth JH. Rosuvastatin: a review of the pharmacology and clinical effectiveness in cardiovascular disease. Clin Med Insights Cardiol. 2012;6:17-33. 
21. Ridker PM. Rosuvastatin in the primary prevention of cardiovascular disease among patients with low levels of low-density lipoprotein cholesterol and elevated high-sensitivity C-reactive protein: rationale and design of the JUPITER trial. Circulation. 2003;108(19):2292-2297.

22. Ridker PM, Danielson E, Fonseca FA, et al; JUPITER Study Group. Rosuvastatin to prevent vascular events in men and women with elevated C-reactive protein. $N$ Engl J Med. 2008;359(21):2195-2207.

23. Sever PS, Dahlöf B, Poulter NR, et al; ASCOT investigators. Prevention of coronary and stroke events with atorvastatin in hypertensive patients who have average or lower-than-average cholesterol concentrations, in the Anglo-Scandinavian cardiac outcomes trial-lipid lowering arm (ASCOT-LLA): a multicentre randomised controlled trial. Lancet. 2003;361(9364):1149-1158.

24. Waters DD, Guyton JR, Herrington DM, McGowan MP, Wenger NK, Shear C. Treating to new targets (TNT) study: does lowering lowdensity lipoprotein cholesterol levels below currently recommended guidelines yield incremental clinical benefit? Am J Cardiol. 2004;93(2): 154-158.

25. Schwartz GG, Olsson AG, Ezekowitz MD, et al; Myocardial Ischemia Reduction with Aggressive Cholesterol Lowering (MIRACL) Study Investigators. Effects of atorvastatin on early recurrent ischemic events in acute coronary syndromes: the MIRACL study: a randomized controlled trial. JAMA. 2001;285(13):1711-1718.

26. Gibson CM, Pride YB, Hochberg CP, Sloan S, Sabatine MS, Cannon CP. Effect of intensive statin therapy on clinical outcomes among patients undergoing percutaneous coronary intervention for acute coronary syndrome. PCI-PROVE IT: a PROVE IT-TIMI 22 (pravastatin or atorvastatin evaluation and infection therapy-thrombolysis in myocardial infarction 22) substudy. J Am Coll Cardiol. 2009;54(24):2290-2295.

27. Jones PH, Davidson MH, Stein EA, et al; STELLAR Study Group. Comparison of the efficacy and safety of rosuvastatin versus atorvastatin, simvastatin, and pravastatin across doses (STELLAR* Trial). Am J Cardiol. 2003;92(2):152-160.

28. Clearfield MB, Amerena J, Bassand JP, et al. Comparison of the efficacy and safety of rosuvastatin $10 \mathrm{mg}$ and atorvastatin $20 \mathrm{mg}$ in high-risk patients with hypercholesterolemia - prospective study to evaluate the use of low doses of the statins atorvastatin and rosuvastatin (PULSAR). Trials. 2006;7:35.

29. Nicholls SJ, Ballantyne CM, Barter PJ, et al. Effect of two intensive statin regimens on progression of coronary disease. $N$ Engl J Med. 2011;365(22):2078-2087.

30. Schlessinger L, Eddy DM. Archimedes: a new model for simulating health care systems - the mathematical formulation. J Biomed Inform. 2002;35(1):37-50.

31. Eddy DM, Schlessinger L. Archimedes: a trial-validated model of diabetes. Diabetes Care. 2003;26(11):3093-3101.

32. MH4MG. Computer modeling of diabetes and its complications: a report on the fourth Mount Hood challenge meeting. Diabetes Care. 2007;30(6):1638-1646.

33. Schlessinger L, Eddy DM. A Predictive Validation of the CARDS Trial. 2011. Available from: https://archimedesmodel.com/tech-reports.

34. Eddy DM, Cohen MD, Shum K, Dziuba J. Validation Methodology and Results: ARCHeS Simulator 2.5. 2013. Available from: https:// archimedesmodel.com/tech-reports.

35. Schuetz CA, Alperin P, Guda S, et al. A standardized vascular disease health check in europe: a cost-effectiveness analysis. PLoS One. 2013;8(7):e66454

36. Samuel S, Peskin B, Arondekar B, et al. Estimating health and economic benefits from using prescription omega- 3 fatty acids in patients with severe hypertriglyceridemia. Am J Cardiol. 2011;108(5): 691-697.

37. Noah-Vanhoucke J, Green LE, Dinh TA, Alperin P, Smith RA. Costeffectiveness of chemoprevention of breast cancer using tamoxifen in a postmenopausal US population. Cancer. 2011;117(15): 3322-3331.

38. Peskin BR, Shcheprov AV, Boye KS, Bruce S, Maggs DG, Gaebler JA. Cardiovascular outcomes associated with a new once-weekly GLP-1 receptor agonist vs traditional therapies for type 2 diabetes: a simulation analysis. Diabetes Obes Metab. 2011;13(10):921-927.
39. CDC. National Health and Nutrition Examination Survey Data 19992008. 2014. Available from: http://www.cdc.gov/nchs/nhanes.htm.

40. Diabetes Prevention Program. Strategies to identify adults at high risk for type 2 diabetes: the diabetes prevention program. Diabetes Care. 2005;28(1):138-144.

41. Colhoun HM, Betteridge DJ, Durrington PN, et al; CARDS investigators. Primary prevention of cardiovascular disease with atorvastatin in type 2 diabetes in the collaborative atorvastatin diabetes study (CARDS): multicentre randomised placebo-controlled trial. Lancet. 2004;364(9435):685-696.

42. Schuetz CA, van Herick A, Alperin P, Peskin B, Hsia J, Gandhi S. Comparing the effectiveness of rosuvastatin and atorvastatin in preventing cardiovascular outcomes: estimates using the Archimedes model. J Med Econ. 2012;15(6):1118-1129.

43. Schuetz A, van Herick A, Alperin P, Peskin B, Hsia J, Gandhi S. Comparing the effectiveness of rosuvastatin and atorvastatin in preventing cardiovascular outcomes: estimates using the Archimedes model. Value Health. 2011;14(3):A36.

44. van Herick A, Schuetz CA, Alperin P, Bullano MF, Balu S, Gandhi S. The impact of initial statin treatment decisions on cardiovascular outcomes in clinical care settings: estimates using the Archimedes model. Clinicoecon Outcomes Res. 2012;4:337-347.

45. Folse H, Sternhufvud C, Andy Schuetz C, Rengarajan B, Gandhi S. Impact of switching treatment from rosuvastatin to atorvastatin on rates of cardiovascular events. Clin Ther. 2014;36(1):58-69.

46. Robinson JG, Smith B, Maheshwari N, Schrott H. Pleiotropic effects of statins: benefit beyond cholesterol reduction? A meta-regression analysis. J Am Coll Cardiol. 2005;46(10):1855-1862.

47. D'Agostino RB Sr, Vasan RS, Pencina MJ, et al. General cardiovascular risk profile for use in primary care: the Framingham heart study. Circulation. 2008;117(6):743-753.

48. Chobanian AV, Bakris GL, Black HR, et al. Seventh report of the joint national committee on prevention, detection, evaluation, and treatment of high blood pressure. Hypertension. 2003;42(6):1206-1252.

49. Nathan DM, Buse JB, Davidson MB, et al. Management of hyperglycemia in type 2 diabetes: a consensus algorithm for the initiation and adjustment of therapy: update regarding thiazolidinediones: a consensus statement from the American diabetes association and the European association for the study of diabetes. Diabetes Care. 2008;31(1): 173-175.

50. Waters DD, Brotons C, Chiang CW, et al; Lipid Treatment Assessment Project 2 Investigators. Lipid treatment assessment project 2: a multinational survey to evaluate the proportion of patients achieving low-density lipoprotein cholesterol goals. Circulation. 2009;120(1): 28-34.

51. Eddy DM, Schlessinger L. Validation of the archimedes diabetes model. Diabetes Care. 2003;26(11):3102-3110.

52. Reiner Z, De Bacquer D, Kotseva K, Prugger C, De Backer G, Wood D. Treatment potential for dyslipidaemia management in patients with coronary heart disease across Europe: findings from the EUROASPIRE III survey. Atherosclerosis. 2013;231(2):300-307.

53. Vanuzzo D, Pilotto L, Ambrosio GB, et al; EUROASPIRE Study Group. Potential for cholesterol lowering in secondary prevention of coronary heart disease in Europe: findings from EUROASPIRE study. European action on secondary prevention through intervention to reduce events. Atherosclerosis. 2000;153(2):505-517.

54. Hermans MP, Castro Cabezas M, Strandberg T, et al. Centralized Pan-European survey on the under-treatment of hypercholesterolaemia (CEPHEUS): overall findings from eight countries. Curr Med Res Opin. 2010;26(2):445-454.

55. Perrone-Filardi P, Poli A, Ambrosio G, Proto C, Chimini C, Chiariello M. Implementation of cardiovascular secondary prevention guidelines in clinical practice: a nationwide survey in Italy. Nutr Metab Cardiovasc Dis. 2012;22(2):149-153.

56. Hobbs FD, Erhardt L. Acceptance of guideline recommendations and perceived implementation of coronary heart disease prevention among primary care physicians in five European countries: the reassessing European attitudes about cardiovascular treatment (REACT) survey. Fam Pract. 2002;19(6):596-604. 
57. Dallongeville J, Banegas JR, Tubach F, et al; EURIKA Investigators. Survey of physicians' practices in the control of cardiovascular risk factors: the EURIKA study. Eur J Cardiovasc Prev Rehabil. 2011;19(3): 541-550.

58. Svilaas A, Risberg K, Thoresen M, Ose L. Lipid treatment goals achieved in patients treated with statin drugs in Norwegian general practice. Am J Cardiol. 2000;86(11):1250-1253, A6.

59. Ruof J, Klein G, Marz W, Wollschlager H, Neiss A, Wehling M. Lipidlowering medication for secondary prevention of coronary heart disease in a German outpatient population: the gap between treatment guidelines and real life treatment patterns. Prev Med. 2002;35(1):48-53.

60. Athyros VG, Papageorgiou AA, Mercouris BR, et al. Treatment with atorvastatin to the national cholesterol educational program goal versus 'usual' care in secondary coronary heart disease prevention. The GREek atorvastatin and coronary-heart-disease evaluation (GREACE) study. Curr Med Res Opin. 2002;18(4):220-228.

61. van Dam M, van Wissen S, Kastelein JJ. Declaring war on undertreatment: rationale for an aggressive approach to lowering cholesterol. J Cardiovasc Risk. 2002;9(2):89-95.

62. Willey VJ, Bullano MF, Shoetan NN, Gandhi SK. Therapy modifications and low-density lipoprotein cholesterol goal attainment rates associated with the initiation of generic simvastatin. Curr Med Res Opin. 2010;26(1):121-128.

63. Simpson RJ Jr, Tunceli K, Ramey DR, et al. Treatment pattern changes in high-risk patients newly initiated on statin monotherapy in a managed care setting. J Clin Lipidol. 2013;7(5):399-407.

64. Jones PH. Statins: the case for higher, individualized starting doses. Cleve Clin J Med. 2005;72(9):811-816.

65. McVey D, Patel H, Eminton Z, Maton S. An assessment of the efficacy of atorvastatin in treating patients with dyslipidaemia to target LDL-cholesterol goals: the atorvastatin matrix study. Int J Clin Pract. 1999;53(7):509-513.

66. Jones PH, McKenney JM, Karalis DG, Downey J. Comparison of the efficacy and safety of atorvastatin initiated at different starting doses in patients with dyslipidemia. Am Heart J. 2005;149(1):e1.
67. Ducobu J, Claeys M, Commers K, et al. Efficacy of atorvastatin after LDL-cholesterol-based dose selection in high risk dyslipidaemic patients: results of the target dose study. Curr Med Res Opin. 2007; 23(8):1821-1827.

68. Farsang C, Athyros V, Gaw A. A multicentre, open study to assess the effect of individualizing starting doses of atorvastatin according to baseline LDL-C levels on achieving cholesterol targets: the achieve cholesterol targets fast with atorvastatin stratified titration (ACTFAST-2) study. Curr Med Res Opin. 2007;23(8):1945-1956.

69. Fox KM, Gandhi SK, Ohsfeldt RL, Blasetto JW, Davidson MH. Titration patterns with rosuvastatin as compared with other statins in clinical practice: a retrospective observational cohort study using an electronic medical record database. Clin Ther. 2007;29(11): 2385-2394.

70. Stargardt T. The impact of reference pricing on switching behaviour and healthcare utilisation: the case of statins in Germany. Eur J Health Econ. 2010;11(3):267-277.

71. Sakshaug S, Furu K, Karlstad O, Ronning M, Skurtveit S. Switching statins in Norway after new reimbursement policy: a nationwide prescription study. Br J Clin Pharmacol. 2007;64(4):476-481.

72. Gizurarson S, Bjornsdottir LR, Einarsdottir R, Halldorsson M, Andersen K. Clinical consequences following regulatory changes in respect to reimbursement of statins cost by the Icelandic Social Insurance Administration. Scand J Public Health. 2012;40(7):663-667.

73. Tunceli K, Sajjan SG, Ramey DR, et al. Switching from high-efficacy lipid-lowering therapies to simvastatin and low-density lipoprotein cholesterol goal attainment in coronary heart disease/coronary heart disease-equivalent patients. J Clin Lipidol. 2010;4(6):491-500.

74. Maningat P, Gordon BR, Breslow JL. How do we improve patient compliance and adherence to long-term statin therapy? Curr Atheroscler Rep. 2013;15(1):291.

75. Conroy RM, Pyörälä K, Fitzgerald AP, et al; SCORE project group. Estimation of ten-year risk of fatal cardiovascular disease in Europe: the SCORE project. Eur Heart J. 2003;24(11):987-1003.
ClinicoEconomics and Outcomes Research

\section{Publish your work in this journal}

ClinicoEconomics \& Outcomes Research is an international, peerreviewed open-access journal focusing on Health Technology Assessment, Pharmacoeconomics and Outcomes Research in the areas of diagnosis, medical devices, and clinical, surgical and pharmacologica intervention. The economic impact of health policy and health systems

\section{Dovepress}

organization also constitute important areas of coverage. The manuscript management system is completely online and includes a very quick and fair peer-review system, which is all easy to use. Visit http://www.dovepress.com/testimonials.php to read real quotes from published authors. 